\title{
Rings of Fractions of $B(H)$
}

By

\author{
Yoshinobu KATO *
}

\section{§ 1. Introduction}

In this paper we discuss the following question : What are rings of fractions of $B(H)$, the algebra of all bounded linear operators on a separable, infinite dimensional, Hilbert space $H$ ? We recall the definition of a ring of fractions of a (generally non-commutative) ring according to [4].

Definition. A subset $S$ of a ring A with a unit 1 is called a (right) denominator set if $S$ satisfies the following conditions :

(S0) If $s, t \in S$, then $s t \in S$, and $1 \in S$.

(S1) If $s \in S$ and $a \in A$, then there exist $t \in S$ and $b \in A$ such that $s b=a t$.

(S2) If $s a=0$ with $s \in S$, then $a t=0$ for some $t \in S$.

(S3) $S$ does not contain $0 . \quad$ (to avoid triviality).

Definition. The ring $A\left[S^{-1}\right]$ of fractions of a ring $A$ with respect to a (right) denominator set $S$ is defined by $A\left[S^{-1}\right]=(A \times S) / \sim$, where $\sim$ is the equivalence relation on $A \times S$ defined as $(a, s) \sim(b, t)$ if there exist $c, d \in A$ such that $a c=b d$ and $s c=t d \in S$. We define addition and multiplication of $(a, s) \sim,(b, t)^{\sim} \in(A \times S) / \sim$ in the obvious way:

$$
\begin{aligned}
& (a, s)^{\sim}+(b, t)^{\sim}=(a c+b d, u)^{\sim} \text { for some } c \in A, u \text { and } d \in S \text { with } u=s c=t d, \\
& (a, s)^{\sim} \cdot(b, t)^{\sim}=(a c, t u)^{\sim} \text { for some } c \in A \text { and } u \in S \text { with } s c=b u .
\end{aligned}
$$

Moreover if $A$ has a scalar (complex number) multiple, then also does $A\left[S^{-1}\right]$. Then $\varphi(a)=(a, 1)^{\sim}$ defines a homomorphism $\varphi: A \rightarrow(A \times S) / \sim=A\left[S^{-1}\right]$.

Communicated by H. Araki, September 30, 1993.

1991 Mathematics Subject Classifications : 47 D 25

* Nagaikeso Institute, 1983, Kanaoka-cho, Sakai city, Osaka 591 Japan. 
Our main theorem asserts that any ring of fractions $B(H)\left[S^{-1}\right]$ is isomorphic to $B(H)$ or the quotient ring $B(H) / J$ of $B(H)$ by the ideal $J$ of finite rank operators. The next problem is the existence of such a denominator set $S$. It clear that $B(H)\left[S^{-1}\right]=B(H)$ if we take $S=\{1\}$. We shall show that there exist at least countably infinite many different denominator sets $S$ such that $B(H)\left[S^{-1}\right]$ are isomorphic to $B(H) / J$.

\section{§ 2. Main Theorem}

An operator $x \in B(H)$ is a Fredholm operator if $\operatorname{ran} x$ is closed, $\operatorname{dim} \operatorname{ker} x$ is finite and $\operatorname{dim} \operatorname{ker} x^{*}$ is finite, where $\operatorname{ran} x$ is the range of $x$ and $\operatorname{ker} x$ is the kernel of $x$. The collection of Fredholm operators is denoted by $F$. The ind is the function from $F$ to the integers $\mathbb{Z}$ defined by ind $x=\operatorname{dim} \operatorname{ker} x-\operatorname{dim} \operatorname{ker} x^{*}$. This function enjoys the following property : For $x, y \in F$, ind $x y=$ ind $x+$ ind $y$, ind $x^{*}=-$ ind $x$, ind $1=0$. Put $F_{0}=\{x \in F \mid$ ind $x=0\}$. Then $F$ and $F_{0}$ satisfy (S0). Moreover $F$ and $F_{0}$ are invariant under compact perturbations ([1]). If $x$ and $y \in B(H)$ satisfy $x y x$ $=x, y x y=y,(x y)^{*}=x y$ and $(y x)^{*}=y x$, then $y$ is called a Moore-Penrose inverse of $x$ and $y$ is denoted by $x^{\dagger}$. A Moore-Penrose inverse $x^{\dagger}$ does not always exist but it is unique if it exists. It is known that $x^{\dagger}$ exists if and only if $\operatorname{ran} x$ is closed ([3]). In particular if $x$ is in $F$, then $x$ has $x^{\dagger}$.

We need the following Theorem in $[2$; Theorem 3.6] :

Theorem $\mathbb{F}-\mathrm{W}$. Let $S$ be in $B(H)$. If ran $s$ is not closed, then there exists a unitary $u \in B(H)$ such that $\operatorname{ran} s \cap \operatorname{ran} u s=\{0\}$.

We shall show that a denominator is automatically a Fredholm operator.

Theorem 1 . If a subset $S \subset B(H)$ is a denominator set of $B(H)$, then $S$ is contained in the set $F$ of Fredholm operators.

Proof. Let $s \in S$. Assume that ran $s$ is not closed. Then by Theorem F-W, there exists a unitary $u$ such that $\operatorname{ran} s \cap \operatorname{ran} u s=\{0\}$. The condition (S1) implies that there exist $t \in S$ and $b \in B(H)$ such that $s b=(u s) t$. Then

$$
\operatorname{ran} u s t=\operatorname{ran} s b=\operatorname{ran} s b \cap \operatorname{ran} u s t \subset \operatorname{ran} s \cap \operatorname{ran} u s=\{0\} .
$$

Therefore $u s t=0$. Then $S$ contains $s t=0$. This contradicts to (S3). Hence ran $s$ is closed. Next assume that $\operatorname{dim} \operatorname{ker} s^{*}=+\infty$. Then there exists a unitary $u$ such that $\operatorname{ran} u \cap \operatorname{ran} u s=\{0\}$, since $\operatorname{dim}(\operatorname{ran} s)^{\perp}=\operatorname{dim} \operatorname{ker} s^{*}=+\infty$. By the same argument of the proceeding paragraph, $S$ contains 0 . This is a contradiction. Therefore $\operatorname{dim} \operatorname{ker} s^{*}<+\infty$. Next we shall show that $\operatorname{dim} \operatorname{ker} s<+\infty$. Since ran $s$ 
is closed, $s^{\dagger}$ exists. Put $a=1-s^{\dagger} s$, then $s a=0$. By (S2) there exists $t \in S$ such that $a t=0$. Since $a=a^{*}, t^{*} a=0$, that is, $\operatorname{ran} a \subset \operatorname{ker} t^{*}$. Then $\operatorname{dim} \operatorname{ran} a \leqq \operatorname{dim} \operatorname{ker} t^{*}<$ $+\infty$, because $t \in S$. Thus $\operatorname{dim} \operatorname{ker} s=\operatorname{dim} \operatorname{ran} a<+\infty$. Therefore $s \in S$ is a Fredholm operator.

Consider the canonical homomorphism $\varphi: B(H) \rightarrow B(H)\left[S^{-1}\right]$ defined by $\varphi(x)=(x, 1)^{\sim}$.

Lemma 2. The canonical map $\varphi: B(H) \rightarrow B(H)\left[S^{-1}\right]$ is onto.

Proof. Take $(a, s)^{\sim} \in B(H)\left[S^{-1}\right]$. Then $s^{\dagger}$ exists by Theorem 1. Put $z=1-$ $s^{\dagger} s$. Since $s z=0$, there exists $c \in S$ such that $z c=0$ by (S2). Then $c=s^{\dagger} s c$. Put $x=$ $a s^{\dagger}$ and $d=s c$. Then

$$
a c=a s^{\dagger} s c=a s^{\dagger} d \in B(H) \text { and } s c=l d \in S .
$$

This shows that $(a, s) \sim\left(a s^{\dagger}, 1\right)$. Then $\varphi(x)=\left(a s^{\dagger}, 1\right)^{\sim}=(a, s) \sim$. Thus $\varphi$ is onto.

The following main theorem gives the possible rings of fractions of $B(H)$ completely :

Theorem 3. Let $S$ be a denominator set of $B(H)$. If $S$ contains a non-invertible operator, then the ring $B(H)\left[S^{-1}\right]$ of fractions is isomorphic to the quotient ring $B(H) / J$ of $B(H)$ by the ideal $J$ of finite rank operators. If $S$ does not, then $B(H)$ $\left[S^{-1}\right]$ is isomorphic to $B(H)$.

Proof. By Lemma 2, $B(H)\left[S^{-1}\right]$ is isomorphic to $B(H) / \operatorname{ker} \varphi$. We note that

(*) $\quad \operatorname{ker} \varphi=\{x \in B(H) \mid x c=0$ for some $c \in S\}$.

If $S$ does not contain non-invertible elements, then $\operatorname{ker} \varphi=\{0\}$, so $B(H)\left[S^{-1}\right]$ is isomorphic to $B(H)$. Now suppose that $S$ contains a non-invertible operator $s$. Then $s^{\dagger} \neq 1$ or $s s^{\dagger} \neq 1$. If $s s^{\dagger} \neq 1$, then $x=1-s s^{\dagger} \neq 0$ and $x \in \operatorname{ker} \varphi$, because $x s=s-$ $s s^{\dagger} s=0$ and $s \in S$. If $s^{\dagger} s \neq 1$, put $x=1-s^{\dagger} s$. Since $s x=0, x t=0$ for some $t \in S$ by (S2). Thus $x \neq 0$ and $x \in \operatorname{ker} \varphi$. In any case we have that $\operatorname{ker} \varphi \neq\{0\}$. Next we shall show that $\operatorname{ker} \varphi \subset J$. Let $x \in \operatorname{ker} \varphi$. By (*) there exists $c \in S$ such that $x c=$ 0 . Since $c^{*} x^{*}=0, \operatorname{ran} x^{*} \subset \operatorname{ker} c^{*}$. By Theorem 1, $c$ is a Fredholm operator and $\operatorname{dim} \operatorname{ker} c^{*}<+\infty$. Hence $x^{*}$ is a finite rank operator, so $x \in J$. Since $J$ is a non-trivial minimal two-sided ideal of $B(H), \operatorname{ker} \varphi=J$. Therefore if $S$ contains a non-invertible element, then $B(H)\left[S^{-1}\right]$ is isomorphic to $B(H) / J$. 


\section{§3. Examples of Denominator Sets}

In this section we shall give some examples of a denominator set $S$ such that

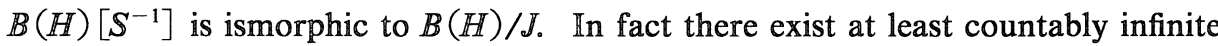
many denominator sets with this property, although we have not yet determined all of them.

Theorem 4. If $S$ is a semigroup such that $F_{0} \subset S \subset F$, then $S$ is a denominator set. In particular $\mathbb{F}_{0}$ and $\mathbb{F}$ are denominator sets.

Proof. It is clear that $S$ satisfies (S0) and (S3). We shall show that $S$ satisfies (S1). Take $s \in S$ and $a \in \mathbb{B}(H)$. Since $s \in F$, $s^{\dagger}$ exists. Then $1-s s^{\dagger} \in J$, because $\operatorname{dim} \operatorname{ran}\left(1-s s^{\dagger}\right)=\operatorname{dim} \operatorname{ker} s^{*}<+\infty$. Put $c=\left(1-s s^{\dagger}\right) a$. Then $c$ is also in $J$, so ran $c$ is closed and $c^{\dagger}$ exists. Then $c^{\dagger} c$ is in $J$. Put $t=1-c^{\dagger} c$. Since $t$ is a compact perturbation of $1, t \in F_{0} \subset \mathbb{S}$. Put $b=s^{\dagger} a t$. Then

$$
a t-s b=\left(1-s s^{\dagger}\right) a t=\left(1-s s^{\dagger}\right) a\left(1-c^{\dagger} c\right)=c\left(1-c^{\dagger} c\right)=0 .
$$

So $s b=a t$. Thus $S$ satisfies (S1). Next we shall show that $S$ satisfies (S2). Take $s$ $\in S$ and $s \in \mathbb{B}(\mathbb{H})$ such that $s a=0$. Since $\operatorname{ran} a \subset \operatorname{ker} s, a$ is in $J$. Consider a polar decomposition $a=u|a|$. We may assume that $u$ is a unitary. Put $t=u^{*} s^{*} s$. Then ind $t=$ ind $u *-$ ind $s+$ ind $s=0$. Hence $t \in F_{0} \subset S$. And $a t=u|a| u{ }^{*}{ }^{*} s=u a{ }^{*}{ }^{*} s=$ $u(s a)^{*} s=0$. Thus $\mathbb{S}$ satisfies $(\mathbb{S} 2)$.

Finally we shall give two kinds of examples of denominator sets of $B(H)$ which do not contain $\mathbb{F}_{0}$. Let $\mathbb{K}$ be a separable, infinite dimensional, Hilbert space and $n$ be a positive integer. Put $H=\mathbb{K} \oplus \cdots \oplus \mathbb{K}$ ( $n$ times). Then $B(H)$ can be identified with the set $M_{n}(B(\mathbb{K}))$ of $n \times n$ matrices whose entries are in $B(K)$. Let $S$ be a denominator set of $B(\mathbb{K})$. Define $S_{n}$ and $S^{n} \subset B(\mathbb{H})$ by

$$
\begin{aligned}
& S_{n}=\left\{\left(\begin{array}{cc}
s & 0 \\
s & \ddots \\
0 & s
\end{array}\right) \in B(H) \mid s \in S\right\}
\end{aligned}
$$

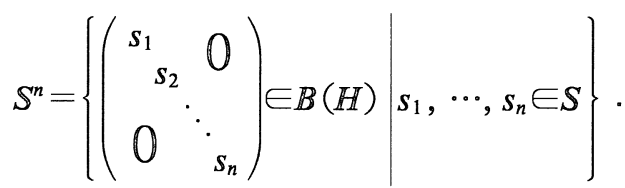

By $[4 ;$ page 61 , Exercises 4$], S_{n}$ is a denominator set of $B(H)$. Similarly we can show that $\mathbb{S}^{n}$ is also a denominator set of $B(H)$. Therefore we get the following: 
Theorem 5. There exist countably infinite many denominator sets $S$ of $B(H)$ such that $B(H)\left[S^{-1}\right]$ are isomorphic to $B(H) / J$.

\section{Acknowledgement}

The author would like to thank Prof. Y. Watatani for his help.

\section{References}

[1] Douglas, R. G., Banach Algebra Technique in Operator Theory, Academic Press, New York, 1972.

[2] Fillmore, P. A. and Williams, J, On operator ranges, Adv. Math., 7 (1971), 254-281.

[3] Groetch, C. W., Generalized Inverses of Linear Operators : Representation and Application, Dekker, New York, 1977.

[ 4 ] Stenström, B., Rings of Quotients, Springer-Verlag, Berlin Heidelberg New York, 1975. 
